\title{
On the Consistency of Multi-robot Cooperative Localization
}

\author{
Guoquan P. Huang*, Nikolas Trawny*, Anastasios I. Mourikis ${ }^{\dagger}$, and Stergios I. Roumeliotis* \\ ${ }^{*}$ Dept. of Computer Science and Engineering, University of Minnesota, Minneapolis, MN 55455 \\ Email: \{ghuang |trawny|stergios\}@cs.umn.edu \\ ${ }^{\dagger}$ Dept. of Electrical Engineering, University of California, Riverside, CA 92521 \\ Email: mourikis@ee.ucr.edu
}

\begin{abstract}
In this paper, we investigate the consistency of extended Kalman filter (EKF)-based cooperative localization (CL) from the perspective of observability. To the best of our knowledge, this is the first work that analytically shows that the error-state system model employed in the standard EKF-based CL always has an observable subspace of higher dimension than that of the actual nonlinear CL system. This results in unjustified reduction of the EKF covariance estimates in directions of the state space where no information is available, and thus leads to inconsistency. To address this problem, we adopt an observabilitybased methodology for designing consistent estimators and propose a novel Observability-Constrained (OC)-EKF. In contrast to the standard EKF-CL, the linearization points of the OC-EKF are selected so as to ensure that the dimension of the observable subspace remains the same as that of the original (nonlinear) system. The proposed OC-EKF has been tested in simulation and experimentally, and has been shown to significantly outperform the standard EKF in terms of both accuracy and consistency.
\end{abstract}

\section{INTRODUCTION}

In order for multi-robot teams to perform tasks such as exploration, surveillance, and search and rescue, their members need to precisely determine their positions and orientations (poses). In GPS-denied areas and in the absence of robust landmarks, teams of robots can still jointly estimate their poses by sharing relative position measurements (cf. [1], [2], [3]). Current approaches to solving the cooperative localization (CL) problem, in either centralized or distributed fashion, are based on the extended Kalman filter (EKF) [3], maximum likelihood estimation (MLE) [4], maximum a posteriori (MAP) estimation [5], or particle filtering (PF) [6]. Among these algorithms, the EKF arguably remains a popular choice due to its relatively low computational cost and easy implementation.

While recent research efforts have focused on reducing the computational complexity of EKF-CL [7], [8], [9], the fundamental issue of consistency has received little attention. As defined in [10], a state estimator is consistent if the estimation errors are zero-mean and have covariance smaller than or equal to the one calculated by the filter. Consistency is one of the primary criteria for evaluating an estimator's performance; if an estimator is inconsistent, then the accuracy of the produced state estimates is unknown, which renders them unreliable.

In this paper, we present a study of the consistency of EKFbased CL from the perspective of observability. Based on the study, we introduce a novel EKF estimator that significantly improves consistency as well as accuracy. In particular, the major contributions of this work are the following:

- We investigate the observability properties of the errorstate system model employed by the EKF, and show that its observable subspace has higher dimension than that of the underlying nonlinear CL system. As a result, the covariance estimates of the EKF undergo reduction in directions of the state space where no information is available, hence leading to inconsistency. To the best of our knowledge, this is the first work to identify and report the inconsistency of EKF-CL.

- Based on the observability analysis, we introduce the Observability-Constrained (OC)-EKF to improve consistency. Specifically, the linearization points of the OC-EKF's system model are judiciously selected to ensure that the linearized CL system has an observable subspace of the same dimension as the nonlinear CL system. We show that this requirement is satisfied by evaluating the state-propagation Jacobians at the state estimates before (instead of after) each update, while the measurement Jacobians are computed in the same way as for the standard EKF. Through extensive simulation and experimental tests, we verify that the OC-EKF outperforms the standard EKF in terms of both accuracy and consistency, even though it uses older (and thus less accurate) state estimates to compute the filter Jacobians. This result in turn indicates that the observability properties of the system model play a key role in determining the filter's accuracy and consistency.

\section{RELATED WORK}

To the best of our knowledge, no work has yet analytically addressed the consistency issue in CL. In contrast, recent research has focused on the consistency of EKF-SLAM (cf. [11], [12], [13], [14], [15]) showing that the computed state estimates tend to be inconsistent. Specifically, Julier and Uhlmann [11] first observed that when a stationary robot measures the relative position of a new landmark multiple times, the estimated variance of the robot's orientation becomes smaller. Since the observation of a previously unseen feature does not provide any information about the robot's state, this reduction is "artificial" and thus leads to inconsistency. Bailey et al. [13] examined several symptoms of the inconsistency of the standard EKF SLAM algorithm, and argued, based on 
simulation results, that the uncertainty in the robot orientation is the main cause of the inconsistency of EKF-SLAM. Huang and Dissanayake [14] extended the analysis of [11] to the case of a robot observing a landmark from two positions (i.e., the robot observes a landmark, moves and then re-observes the landmark), and proposed a constraint that the filter Jacobians must satisfy to allow for consistent estimation. They also showed that this condition is generally violated, due to the fact that the filter Jacobians at different time instants are computed using different estimates for the same state variables.

In our previous work [15], we conducted a theoretical analysis of EKF-SLAM inconsistency and identified as a fundamental cause the mismatch in the dimensions of the observable subspaces between the linearized system, employed by the EKF, and the underlying nonlinear system. Furthermore, we introduced the First Estimates Jacobian (FEJ)-EKF, which significantly outperforms the standard EKF and the robocentric mapping algorithm [12], in terms of both accuracy and consistency. The FEJ-EKF solution to the SLAM inconsistency problem was reached by imposing constraints inferred from the system observability analysis, and can serve as a model for a new methodology for designing consistent estimators for nonlinear systems. Such an approach is therefore employed in this work for addressing the inconsistency of EKF-CL.

We should note that a recent publication by Bahr et al. [16] addresses a related but different problem, namely the consistency of a distributed CL algorithm due to re-use of information. In the decentralized estimation scheme of [16], the cross-correlations between the state estimates of different robots are not estimated. However, it is well-known that if cross-correlations between robots are not properly taken into account during filter updates, inconsistency can arise [6], [17]. The algorithm in [16] avoids inconsistency by maintaining a careful record of past robot-to-robot measurement updates. In contrast to the above fully decentralized scenario, in our work the cross-correlation terms are maintained in the filter, and the EKF employed for estimation is optimal, except for the inaccuracies introduced by linearization. Our work focuses on identifying and addressing the cause of inconsistency of this EKF-based CL estimator.

\section{STANDARD EKF-BASED CL}

In this section, we present the equations of 2D EKF-CL with general system and measurement models. ${ }^{1}$ In particular, in the standard formulation of CL, the state vector comprises the $N$ robot poses expressed in the global frame of reference. Thus, at time-step $k$ the state vector is given by:

$$
\mathbf{x}_{k}=\left[\begin{array}{lll}
\mathbf{x}_{1_{k}}^{T} & \ldots & \mathbf{x}_{N_{k}}^{T}
\end{array}\right]^{T}
$$

where $\mathbf{x}_{i_{k}} \triangleq\left[\begin{array}{ll}\mathbf{p}_{i_{k}}^{T} & \phi_{i_{k}}\end{array}\right]^{T}$ denotes the $i$ th robot pose (position and orientation). In general, EKF-CL recursively evolves in two steps: propagation and update, based on the discrete-time system and measurement models, respectively.

\footnotetext{
${ }^{1}$ For the purpose of the consistency study and in order to simplify the derivations, in this paper we focus on centralized EKF-CL. Note that a distributed implementation [3] does not alter the system properties.
}

\section{A. EKF Propagation}

In the propagation step, each robot processes its odometry measurements to obtain an estimate of the pose change between two consecutive time steps, which is then employed in the EKF to propagate the robot state estimate. The EKF propagation equations are given by: ${ }^{2}$

$$
\begin{aligned}
\hat{\mathbf{p}}_{i_{k+1 \mid k}} & =\hat{\mathbf{p}}_{i_{k \mid k}}+\mathbf{C}\left(\hat{\phi}_{i_{k \mid k}}\right) \cdot{ }^{k} \hat{\mathbf{p}}_{i_{k+1}} \\
\hat{\phi}_{i_{k+1 \mid k}} & =\hat{\phi}_{i_{k \mid k}}+{ }^{k} \hat{\phi}_{i_{k+1}}
\end{aligned}
$$

for all $i=1, \ldots, N$. In the above expressions, $\mathbf{C}(\cdot)$ denotes the $2 \times 2$ rotation matrix, and ${ }^{k} \hat{\mathbf{x}}_{i_{k+1}} \triangleq\left[{ }^{k} \hat{\mathbf{p}}_{i_{k+1}}^{T}{ }^{k} \hat{\phi}_{i_{k+1}}\right]^{T}$ is the odometry-based estimate of the $i$ th robot's motion between time-steps $k$ and $k+1$, expressed with respect to the robot's frame of reference at time-step $k$. This estimate is corrupted by zero-mean, white Gaussian noise $\mathbf{w}_{i_{k}}={ }^{k} \mathbf{x}_{i_{k+1}}-{ }^{k} \hat{\mathbf{x}}_{i_{k+1}}$, with covariance $\mathbf{Q}_{k}$. Clearly this system model is nonlinear, and can be described by the following generic nonlinear function:

$$
\mathbf{x}_{i_{k+1}}=\mathbf{f}\left(\mathbf{x}_{i_{k}},{ }^{k} \hat{\mathbf{x}}_{i_{k+1}}+\mathbf{w}_{i_{k}}\right)
$$

In addition to the state propagation equations, the linearized error-state propagation equation is necessary for the EKF:

$$
\tilde{\mathbf{x}}_{i_{k+1 \mid k}}=\boldsymbol{\Phi}_{i_{k}} \tilde{\mathbf{x}}_{i_{k \mid k}}+\mathbf{G}_{i_{k}} \mathbf{w}_{i_{k}}
$$

In the above expression, $\boldsymbol{\Phi}_{i_{k}}$ and $\mathbf{G}_{i_{k}}$ are obtained by linearization of the state propagation equations (2)-(3):

$$
\begin{aligned}
\boldsymbol{\Phi}_{i_{k}} & =\left[\begin{array}{cc}
\mathbf{I}_{2} & \mathbf{J}\left(\hat{\mathbf{p}}_{i_{k+1 \mid k}}-\hat{\mathbf{p}}_{i_{k \mid k}}\right) \\
\mathbf{0}_{1 \times 2} & 1
\end{array}\right] \\
\mathbf{G}_{i_{k}} & =\left[\begin{array}{cc}
\mathbf{C}\left(\hat{\phi}_{i_{k \mid k}}\right) & \mathbf{0}_{2 \times 1} \\
\mathbf{0}_{1 \times 2} & 1
\end{array}\right]
\end{aligned}
$$

where we have employed the identity $\mathbf{C}\left(\hat{\phi}_{i_{k \mid k}}\right) \cdot{ }^{k} \hat{\mathbf{p}}_{i_{k+1}}=$ $\hat{\mathbf{p}}_{i_{k+1 \mid k}}-\hat{\mathbf{p}}_{i_{k \mid k}}$, and $\mathbf{J} \triangleq\left[\begin{array}{cc}0 & -1 \\ 1 & 0\end{array}\right]$.

By stacking all $N$ robots' states to create the state vector for the entire system, we have

$$
\begin{aligned}
& \tilde{\mathbf{x}}_{k+1 \mid k} \\
& =\left[\begin{array}{ccc}
\boldsymbol{\Phi}_{1_{k}} & \cdots & \mathbf{0} \\
\vdots & \ddots & \vdots \\
\mathbf{0} & \cdots & \boldsymbol{\Phi}_{N_{k}}
\end{array}\right]\left[\begin{array}{c}
\tilde{\mathbf{x}}_{1_{k \mid k}} \\
\vdots \\
\tilde{\mathbf{x}}_{N_{k \mid k}}
\end{array}\right]+\left[\begin{array}{ccc}
\mathbf{G}_{1_{k}} & \cdots & \mathbf{0} \\
\vdots & \ddots & \vdots \\
\mathbf{0} & \cdots & \mathbf{G}_{N_{k}}
\end{array}\right]\left[\begin{array}{c}
\mathbf{w}_{1_{k}} \\
\vdots \\
\mathbf{w}_{N_{k}}
\end{array}\right] \\
& \triangleq \boldsymbol{\Phi}_{k} \tilde{\mathbf{x}}_{k \mid k}+\mathbf{G}_{k} \mathbf{w}_{k}
\end{aligned}
$$

It is important to point out that the form of the propagation equations presented above is general, and holds for any robot kinematic model (e.g., unicycle, bicycle, or Ackerman).

\footnotetext{
${ }^{2}$ Throughout this paper the subscript $\ell \mid k$ refers to the estimate of a quantity at time-step $\ell$, after all measurements up to time-step $k$ have been processed, while the superscript $(i j)$ refers to the relative measurement from robot $i$ to robot $j . \hat{x}$ is used to denote the estimate of a random variable $x$, while $\tilde{x}=x-\hat{x}$ is the error in this estimate. $\mathbf{0}_{m \times n}$ and $\mathbf{1}_{m \times n}$ denote $m \times n$ matrices of zeros and ones, respectively, while $\mathbf{I}_{n}$ is the $n \times n$ identity matrix.
} 


\section{B. EKF Update}

The measurements used for updates in CL are always a function of the relative pose of the observed robot $j$ with respect to the observing robot $i$ :

$$
\mathbf{z}_{k}^{(i j)}=\mathbf{h}\left(\mathbf{x}_{i_{k}}, \mathbf{x}_{j_{k}}\right)+\mathbf{v}_{k}^{(i j)}=\mathbf{h}\left({ }^{i} \mathbf{x}_{j_{k}}\right)+\mathbf{v}_{k}^{(i j)}
$$

where ${ }^{i} \mathbf{x}_{j_{k}} \triangleq\left[\begin{array}{c}{ }^{i} \mathbf{p}_{j_{k}} \\ { }^{i} \phi_{j_{k}}\end{array}\right]=\left[\begin{array}{c}\mathbf{C}^{T}\left(\phi_{i_{k}}\right)\left(\mathbf{p}_{j_{k}}-\mathbf{p}_{i_{k}}\right) \\ \phi_{j_{k}}-\phi_{i_{k}}\end{array}\right]$ is the relative pose of the observed robot $j$ with respect to the observing robot $i$ at time-step $k$, and $\mathbf{v}_{k}^{(i j)}$ is zero-mean Gaussian noise with covariance $\mathbf{R}_{k}^{(i j)}$ associated with the measurement $\mathbf{z}_{k}^{(i j)}$. In this work, we allow $\mathbf{h}$ to be any measurement function. For instance, $\mathbf{z}_{k}^{(i j)}$ can be a direct measurement of relative pose, a pair of distance and bearing measurements, bearing-only measurements from monocular cameras, etc. In general, the measurement function is nonlinear, and hence it is linearized for use in the EKF. The linearized measurement-error equation is given by:

$$
\begin{aligned}
& \tilde{\mathbf{z}}_{k}^{(i j)} \simeq\left[\begin{array}{lllllll}
\mathbf{0} & \cdots & \mathbf{H}_{i_{k}}^{(i j)} & \cdots & \mathbf{H}_{j_{k}}^{(i j)} & \cdots & \mathbf{0}
\end{array}\right] \tilde{\mathbf{x}}_{k \mid k-1}+\mathbf{v}_{k}^{(i j)} \\
& \triangleq \mathbf{H}_{k}^{(i j)} \tilde{\mathbf{x}}_{k \mid k-1}+\mathbf{v}_{k}^{(i j)}
\end{aligned}
$$

where $\mathbf{H}_{i_{k}}^{(i j)}$ and $\mathbf{H}_{j_{k}}^{(i j)}$ are the Jacobians of $\mathbf{h}$ with respect to the $i$ th and $j$ th robot poses, respectively, evaluated at the state estimate $\hat{\mathbf{x}}_{k \mid k-1}$. Using the chain rule of differentiation, these are computed as:

$\mathbf{H}_{i_{k}}^{(i j)}=-\left(\nabla \mathbf{h}_{k}^{(i j)}\right) \mathbf{A}\left(\hat{\phi}_{i_{k \mid k-1}}\right)\left[\begin{array}{cc}\mathbf{I}_{2} & \mathbf{J}\left(\hat{\mathbf{p}}_{j_{k \mid k-1}}-\hat{\mathbf{p}}_{i_{k \mid k-1}}\right) \\ \mathbf{0}_{1 \times 2} & 1\end{array}\right]$

$\mathbf{H}_{j_{k}}^{(i j)}=\left(\nabla \mathbf{h}_{k}^{(i j)}\right) \mathbf{A}\left(\hat{\phi}_{i_{k \mid k-1}}\right)$

where $\mathbf{A}\left(\hat{\phi}_{i_{k \mid k-1}}\right) \triangleq\left[\begin{array}{cc}\mathbf{C}^{T}\left(\hat{\phi}_{i_{k \mid k-1}}\right) & \mathbf{0}_{2 \times 1} \\ \mathbf{0}_{1 \times 2} & 1\end{array}\right]$, and $\nabla \mathbf{h}_{k}^{(i j)}$ denotes the Jacobian of $\mathbf{h}$ with respect to the vector ${ }^{i} \mathbf{x}_{j_{k}}$ evaluated at the state estimate $\hat{\mathbf{x}}_{k \mid k-1}$.

\section{CL ObSeRVABILITy ANALysis}

In this section, we perform an observability analysis for the EKF-CL system derived in the previous section, and compare its observability properties with those of the underlying nonlinear system. Based on this analysis, we draw conclusions about the consistency of the filter.

Martinelli and Siegwart [18] have shown that the underlying nonlinear system of CL in general has three unobservable degrees of freedom, corresponding to the global position and orientation. Thus, when the EKF is used for state estimation in CL, we would expect that the system model employed by the EKF also shares this property. However, in this section we show that this is not the case, since the unobservable subspace of the linearized error-state model of the standard EKF is generally only of dimension two.

Note that, in general, the Jacobian matrices $\boldsymbol{\Phi}_{k}, \mathbf{G}_{k}$, and $\mathbf{H}_{k}$ used in the EKF-CL linearized error-state model (cf. (8) and (10)) are defined as:

$$
\mathbf{\Phi}_{k}=\left.\nabla_{\mathbf{x}_{k}} \mathbf{f}\right|_{\left\{\mathbf{x}_{k}^{\star}, \mathbf{0}\right\}}, \quad \mathbf{G}_{k}=\left.\nabla_{\mathbf{w}_{k}} \mathbf{f}\right|_{\left\{\mathbf{x}_{k}^{\star}, \mathbf{0}\right\}}, \mathbf{H}_{k}=\left.\nabla_{\mathbf{x}_{k}} \mathbf{h}\right|_{\mathbf{x}_{k}^{\star}}
$$

In these expressions, $\mathbf{x}_{k}^{\star}$ denotes the linearization point for the state $\mathbf{x}_{k}$, used for evaluating the Jacobians, while a linearization point equal to the zero vector is chosen for the noise. The EKF employs the above linearized system model for propagating and updating the estimates of the covariance matrix, and thus the observability properties of this model affect the performance of the estimator.

The observability properties of the linearized error-state model of EKF-CL can be studied by examining the observability matrix for the time interval between time-steps $k_{o}$ and $k_{o}+m$, defined as [19]:

$$
\begin{aligned}
\mathbf{M} & \triangleq\left[\begin{array}{c}
\mathbf{H}_{k_{o}} \\
\mathbf{H}_{k_{o}+1} \mathbf{\Phi}_{k_{o}} \\
\vdots \\
\mathbf{H}_{k_{o}+m} \boldsymbol{\Phi}_{k_{o}+m-1} \cdots \mathbf{\Phi}_{k_{o}}
\end{array}\right] \\
& =\mathbf{M}\left(\mathbf{x}_{k_{o}}^{\star}, \mathbf{x}_{k_{o}+1}^{\star}, \ldots, \mathbf{x}_{k_{o}+m}\right)
\end{aligned}
$$

The last expression makes explicit the fact that the observability matrix is a function of the linearization points used in computing all the Jacobians within the time interval $\left[k_{o}, k_{o}+m\right]$. In turn, this implies that the choice of linearization points affects the observability properties of the linearized error-state system of the EKF. This key fact is the basis of our analysis. In what follows, we discuss different possible choices for linearization, and the observability properties of the corresponding linearized systems.

\section{A. Ideal EKF-CL}

Before considering the rank of the matrix $\mathbf{M}$, which is constructed using the estimated values of the state in the filter Jacobians, it is interesting to study the observability properties of the "oracle", or "ideal" EKF (i.e., the filter whose Jacobians are evaluated using the true values of the state variables). In the following, all matrices evaluated using the true state values are denoted by the symbol " $\backsim$ ".

For our derivations, it will be useful to define:

$$
\delta \mathbf{p}_{i j}(k, \ell) \triangleq \mathbf{p}_{i_{k}}-\mathbf{p}_{j_{\ell}}
$$

which is the difference between two robot positions at timesteps $k$ and $\ell$. Using the above definition, we note that (cf. (6))

$$
\breve{\mathbf{\Phi}}_{i_{k_{o}+1}} \breve{\mathbf{\Phi}}_{i_{k_{o}}}=\left[\begin{array}{cc}
\mathbf{I}_{2} & \mathbf{J} \boldsymbol{\delta} \mathbf{p}_{i i}\left(k_{o}+2, k_{o}\right) \\
\mathbf{0}_{1 \times 2} & 1
\end{array}\right]
$$

Based on this identity, it is easy to show by induction that

$$
\breve{\mathbf{\Phi}}_{i_{k_{o}+\ell-1}} \breve{\mathbf{\Phi}}_{i_{k_{o}+\ell-2}} \cdots \breve{\mathbf{\Phi}}_{i_{k_{o}}}=\left[\begin{array}{cc}
\mathbf{I}_{2} & \mathbf{J} \boldsymbol{\delta} \mathbf{p}_{i i}\left(k_{o}+\ell, k_{o}\right) \\
\mathbf{0}_{1 \times 2} & 1
\end{array}\right]
$$

which holds for all $\ell>0$.

In the ensuing analysis, we study the case of a two-robot team, and assume that both robots continuously observe each other during the time interval $\left[k_{o}, k_{o}+m\right]$. Note that this is not a necessary assumption and is made only to simplify the notation. The generalized analysis for the case where the team consists of an arbitrary number of robots is presented in [20]. 
The measurement Jacobian $\breve{\mathbf{H}}_{k_{o}+\ell}$ in this case can be written as (cf. (11) and (12)):

$$
\begin{gathered}
\breve{\mathbf{H}}_{k_{o}+\ell}=-\operatorname{Diag}\left(\left(\nabla \breve{\mathbf{h}}_{k_{o}+\ell}^{(12)}\right) \mathbf{A}\left(\phi_{1_{k_{o}+\ell}}\right),\left(\nabla \breve{\mathbf{h}}_{k_{o}+\ell}^{(21)}\right) \mathbf{A}\left(\phi_{2_{k_{o}+\ell}}\right)\right) \\
\times\left[\begin{array}{cccc}
\mathbf{I}_{2} & \mathbf{J} \boldsymbol{\delta} \mathbf{p}_{21}\left(k_{o}+\ell, k_{o}+\ell\right) & -\mathbf{I}_{2} & \mathbf{0}_{2 \times 1} \\
\mathbf{0}_{1 \times 2} & 1 & \mathbf{0}_{1 \times 2} & -1 \\
-\mathbf{I}_{2} & \mathbf{0}_{2 \times 1} & \mathbf{I}_{2} & \mathbf{J} \boldsymbol{\delta} \mathbf{p}_{12}\left(k_{o}+\ell, k_{o}+\ell\right) \\
\mathbf{0}_{1 \times 2} & -1 & \mathbf{0}_{1 \times 2} & 1
\end{array}\right]
\end{gathered}
$$

while the following identity is immediate (cf. (8) and (18)):

$$
\begin{aligned}
& \breve{\mathbf{\Phi}}_{k_{o}+\ell-1} \breve{\boldsymbol{\Phi}}_{k_{o}+\ell-2} \cdots \breve{\boldsymbol{\Phi}}_{k_{o}}= \\
& {\left[\begin{array}{cccc}
\mathbf{I}_{2} & \mathbf{J} \boldsymbol{\delta} \mathbf{p}_{11}\left(k_{o}+\ell, k_{o}\right) & \mathbf{0}_{2 \times 2} & \mathbf{0}_{2 \times 1} \\
\mathbf{0}_{1 \times 2} & 1 & \mathbf{0}_{1 \times 2} & 0 \\
\mathbf{0}_{2 \times 2} & \mathbf{0}_{2 \times 1} & \mathbf{I}_{2} & \mathbf{J} \boldsymbol{\delta} \mathbf{p}_{22}\left(k_{o}+\ell, k_{o}\right) \\
\mathbf{0}_{1 \times 2} & 0 & \mathbf{0}_{1 \times 2} & 1
\end{array}\right]}
\end{aligned}
$$

Multiplication of equations (19) and (20) yields:

$$
\begin{aligned}
& \breve{\mathbf{H}}_{k_{o}+\ell} \breve{\boldsymbol{\Phi}}_{k_{o}+\ell-1} \breve{\boldsymbol{\Phi}}_{k_{o}+\ell-2} \cdots \breve{\boldsymbol{\Phi}}_{k_{o}}= \\
& -\operatorname{Diag}\left(\left(\nabla \breve{\mathbf{h}}_{k_{o}+\ell}^{(12)}\right) \mathbf{A}\left(\phi_{1_{k_{o}+\ell}}\right),\left(\nabla \breve{\mathbf{h}}_{k_{o}+\ell}^{(21)}\right) \mathbf{A}\left(\phi_{2_{k_{o}+\ell}}\right)\right) \times \\
& {\left[\begin{array}{cccc}
\mathbf{I}_{2} & \mathbf{J} \boldsymbol{\delta} \mathbf{p}_{21}\left(k_{o}+\ell, k_{o}\right) & -\mathbf{I}_{2} & -\mathbf{J} \boldsymbol{\delta} \mathbf{p}_{22}\left(k_{o}+\ell, k_{o}\right) \\
\mathbf{0}_{1 \times 2} & 1 & \mathbf{0}_{1 \times 2} & -1 \\
-\mathbf{I}_{2} & -\mathbf{J} \boldsymbol{\delta} \mathbf{p}_{11}\left(k_{o}+\ell, k_{o}\right) & \mathbf{I}_{2} & \mathbf{J} \boldsymbol{\delta} \mathbf{p}_{12}\left(k_{o}+\ell, k_{o}\right) \\
\mathbf{0}_{1 \times 2} & -1 & \mathbf{0}_{1 \times 2} & 1
\end{array}\right]}
\end{aligned}
$$

Using this result, the observability matrix of the ideal EKFCL, $\breve{M}$, can be obtained as (cf. (14)):

$$
\begin{aligned}
& \breve{\mathbf{M}}=\underbrace{-\operatorname{Diag}\left(\left(\nabla \breve{\mathbf{h}}_{k_{o}}^{(12)}\right) \mathbf{A}\left(\phi_{1_{k_{o}}}\right), \cdots,\left(\nabla \breve{\mathbf{h}}_{k_{o}+m}^{(21)}\right) \mathbf{A}\left(\phi_{2_{k_{o}+m}}\right)\right)}_{\breve{\mathbf{D}}} \times \\
& {\left[\begin{array}{rrrr}
\mathbf{I}_{2} & \mathbf{J} \boldsymbol{\delta} \mathbf{p}_{21}\left(k_{o}, k_{o}\right) & -\mathbf{I}_{2} & \mathbf{0}_{2 \times 1} \\
\mathbf{0}_{1 \times 2} & 1 & \mathbf{0}_{1 \times 2} & -1 \\
-\mathbf{I}_{2} & \mathbf{0}_{2 \times 1} & \mathbf{I}_{2} & \mathbf{J} \boldsymbol{\delta} \mathbf{p}_{12}\left(k_{o}, k_{o}\right) \\
\mathbf{0}_{1 \times 2} & -1 & \mathbf{0}_{1 \times 2} & 1
\end{array}\right]}
\end{aligned}
$$

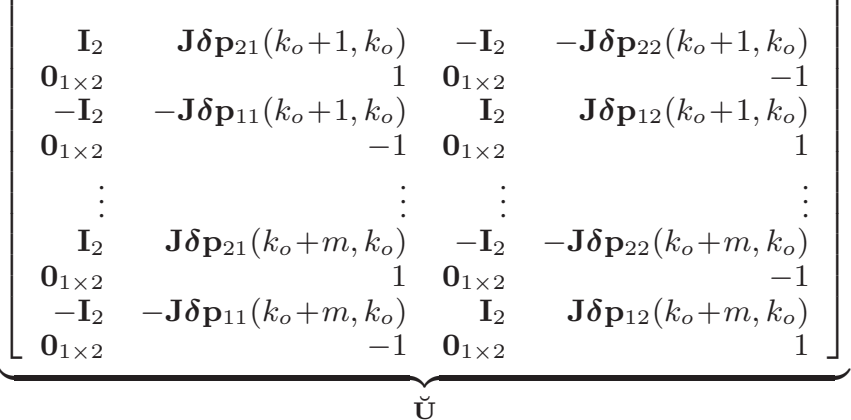

Lemma 4.1: The rank of the observability matrix, $\breve{M}$, of the ideal EKF-CL is equal to 3 .

Proof: The rank of the product of the matrices $\breve{\mathrm{D}}$ and $\breve{\mathbf{U}}$ is given by (cf. (4.5.1) in [21]), $\operatorname{rank}(\breve{\mathbf{D}} \breve{\mathbf{U}})=\operatorname{rank}(\breve{\mathbf{U}})-$ $\operatorname{dim}(\mathcal{N}(\breve{\mathbf{D}}) \bigcap \mathcal{R}(\breve{\mathbf{U}}))$. By denoting $\breve{\mathbf{U}} \triangleq\left[\begin{array}{lll}\breve{\mathbf{u}}_{1} & \cdots & \breve{\mathbf{u}}_{6}\end{array}\right]$, it is evident that $\breve{\mathbf{u}}_{1}=-\breve{\mathbf{u}}_{4}, \breve{\mathbf{u}}_{2}=-\breve{\mathbf{u}}_{5}$, while $\breve{\mathbf{u}}_{3}+\breve{\mathbf{u}}_{6}=$ $\alpha_{1} \breve{\mathbf{u}}_{4}+\alpha_{2} \breve{\mathbf{u}}_{5}$, where $\left[\begin{array}{ll}\alpha_{1} & \alpha_{2}\end{array}\right]^{T} \triangleq-\mathbf{J} \delta \mathbf{p}_{21}\left(k_{o}, k_{o}\right)$. We also note that $\left\{\breve{\mathbf{u}}_{i}\right\}_{i=4}^{6}$ are linearly independent. Therefore, $\mathcal{R}(\breve{\mathbf{U}})=\operatorname{span}\left[\begin{array}{lll}\breve{\mathbf{u}}_{4} & \breve{\mathbf{u}}_{5} & \breve{\mathbf{u}}_{6}\end{array}\right]$. Thus, $\operatorname{rank}(\breve{\mathbf{U}})=3$. We now observe that in general $\breve{\mathbf{D}} \mathbf{u}_{i} \neq \mathbf{0}$, for $i=4,5,6$. Moreover any vector $\mathbf{y} \in \mathcal{R}(\breve{\mathbf{U}}) \backslash \mathbf{0}$ can be written as $\mathbf{y}=\beta_{4} \breve{\mathbf{u}}_{4}+\beta_{5} \breve{\mathbf{u}}_{5}+\beta_{6} \breve{\mathbf{u}}_{6}$ for some $\beta_{i} \in \mathbb{R}$, where $\beta_{i}$, $i=4,5,6$, are not simultaneously equal to zero. Thus, in general, $\breve{\mathbf{D}} \mathbf{y}=\beta_{4} \breve{\mathbf{D}} \breve{\mathbf{u}}_{4}+\beta_{5} \breve{\mathrm{D}}_{5}+\beta_{6} \breve{\mathbf{D}}_{5} \neq \mathbf{0}$, which implies that $\mathbf{y}$ does not belong to the nullspace of $\breve{\mathbf{D}}$. Therefore, $\operatorname{dim}(\mathcal{N}(\breve{\mathbf{D}}) \bigcap \mathcal{R}(\breve{\mathbf{U}}))=0$, and, finally, $\operatorname{rank}(\breve{\mathbf{M}})=$ $\operatorname{rank}(\breve{\mathbf{U}})-\operatorname{dim}(\mathcal{N}(\breve{\mathbf{D}}) \bigcap \mathcal{R}(\breve{\mathbf{U}}))=\operatorname{rank}(\breve{\mathbf{U}})=3$.

Most importantly, by inspection, it can be verified that a basis for the right nullspace of $\mathbf{U}$ (and thus for the right nullspace of $\breve{\mathbf{M}}$ ) is given by:

$$
\mathcal{N}(\breve{\mathbf{M}})=\operatorname{span}\left[\begin{array}{cc}
\mathbf{I}_{2} & \mathbf{J} \mathbf{p}_{1_{k_{o}}} \\
\mathbf{0}_{1 \times 2} & 1 \\
\mathbf{I}_{2} & \mathbf{J} \mathbf{p}_{2_{k_{o}}} \\
\mathbf{0}_{1 \times 2} & 1
\end{array}\right] \triangleq \operatorname{span}\left[\begin{array}{lll}
\mathbf{n}_{1} & \mathbf{n}_{2} & \mathbf{n}_{3}
\end{array}\right]
$$

From the structure of the vectors $\mathbf{n}_{1}$ and $\mathbf{n}_{2}$ we see that a change in the state by $\Delta \mathbf{x}=\alpha \mathbf{n}_{1}+\beta \mathbf{n}_{2}, \alpha, \beta \in \mathbb{R}$ corresponds to shifting the $x-y$ plane by $\alpha$ units along $x$, and by $\beta$ units along $y$. Thus, if the two robots are shifted equally, the states $\mathbf{x}$ and $\mathbf{x}^{\prime}=\mathbf{x}+\Delta \mathbf{x}$ will be indistinguishable given the relative measurements. To understand the physical meaning of $\mathbf{n}_{3}$, we consider the case where the $x-y$ plane is rotated by a small angle $\delta \phi$. Rotating the coordinate system transforms any point $\mathbf{p}=\left[\begin{array}{ll}x & y\end{array}\right]^{T}$ to a point $\mathbf{p}^{\prime}=\left[\begin{array}{ll}x^{\prime} & y^{\prime}\end{array}\right]^{T}$, given by:

$$
\left[\begin{array}{l}
x^{\prime} \\
y^{\prime}
\end{array}\right]=\mathbf{C}(\delta \phi)\left[\begin{array}{l}
x \\
y
\end{array}\right] \simeq\left[\begin{array}{cc}
1 & -\delta \phi \\
\delta \phi & 1
\end{array}\right]\left[\begin{array}{l}
x \\
y
\end{array}\right]=\left[\begin{array}{l}
x \\
y
\end{array}\right]+\delta \phi\left[\begin{array}{c}
-y \\
x
\end{array}\right]
$$

where we have employed the small angle approximations $\cos (\delta \phi) \simeq 1$ and $\sin (\delta \phi) \simeq \delta \phi$. Using this result, we see that if the plane containing the two robots is rotated by $\delta \phi$, the CL state vector will change to $\mathbf{x}^{\prime} \simeq \mathbf{x}+\delta \phi \mathbf{n}_{3}$, which indicates that $\mathbf{n}_{3}$ corresponds to rotation of the $x-y$ plane. This result implies that any such global rotation is unobservable, and will cause no change to the measurements. The preceding analysis for the meaning of the basis vectors of the unobservable subspace agrees with [18] as well as with intuition, which dictates that the global coordinates of the robots (rotation and translation) are unobservable, since the measurements only depend on the relative robot configurations.

\section{B. Standard EKF-CL}

We now study the observability properties of the standard EKF-CL, in which the Jacobians are evaluated at the estimated state (i.e., $\mathbf{x}_{k}^{\star}$ is the latest state estimate). The following definitions will be useful for our derivations:

$$
\begin{aligned}
\mathbf{d} \hat{\mathbf{p}}_{i}(k) \triangleq \hat{\mathbf{p}}_{i_{k \mid k}}-\hat{\mathbf{p}}_{i_{k \mid k-1}} \\
\Delta \hat{\mathbf{p}}_{i j}(k, \ell) \triangleq \hat{\mathbf{p}}_{i_{k \mid k-1}}-\hat{\mathbf{p}}_{j_{k_{o} \mid k_{o}-1}}-\sum_{\kappa=k_{o}}^{\ell} \mathbf{d} \hat{\mathbf{p}}_{j}(\kappa) \\
\delta \hat{\mathbf{p}}_{i j}(k, \ell) \triangleq \hat{\mathbf{p}}_{i_{k \mid k-1}}-\hat{\mathbf{p}}_{j_{\ell \mid \ell-1}}
\end{aligned}
$$

where $k_{o}$ is the first time instant of interest, and $k, \ell \geq k_{o}$. In the above expressions, $\mathbf{d} \hat{\mathbf{p}}_{i}$ is the correction in the $i$ th robot position estimate due to the EKF update at time-step $k$, while $\delta \hat{\mathbf{p}}_{i j}$ is the estimated difference between two robot positions (cf. (16)) evaluated using the uncorrected estimates immediately after the respective propagation steps.

We start by deriving an expression analogous to that of (17):

$$
\boldsymbol{\Phi}_{i_{k_{o}+1}} \boldsymbol{\Phi}_{i_{k_{o}}}=\left[\begin{array}{cc}
\mathbf{I}_{2} & \mathbf{J} \boldsymbol{\Delta} \hat{\mathbf{p}}_{i i}\left(k_{o}+2, k_{o}+1\right) \\
\mathbf{0}_{1 \times 2} & 1
\end{array}\right]
$$


Using induction, we can show that:

$$
\boldsymbol{\Phi}_{i_{k_{o}+\ell-1}} \boldsymbol{\Phi}_{i_{k_{o}+\ell-2}} \cdots \boldsymbol{\Phi}_{i_{k_{o}}}=\left[\begin{array}{cc}
\mathbf{I}_{2} & \mathbf{J} \Delta \hat{\mathbf{p}}_{i i}\left(k_{o}+\ell, k_{o}+\ell-1\right) \\
\mathbf{0}_{1 \times 2} & 1
\end{array}\right]
$$

for $\ell>0$. As a result, the following identity is immediate:

$$
\begin{aligned}
& \boldsymbol{\Phi}_{k_{o}+\ell-1} \boldsymbol{\Phi}_{k_{o}+\ell-2} \cdots \boldsymbol{\Phi}_{k_{o}}= \\
& {\left[\begin{array}{cccc}
\mathbf{I}_{2} & \mathbf{J} \boldsymbol{\Delta} \hat{\mathbf{p}}_{11}\left(k_{o}+\ell, k_{o}+\ell-1\right) & \mathbf{0}_{2 \times 2} & \mathbf{0}_{2 \times 1} \\
\mathbf{0}_{1 \times 2} & 1 & \mathbf{0}_{1 \times 2} & 0 \\
\mathbf{0}_{2 \times 2} & \mathbf{0}_{2 \times 1} & \mathbf{I}_{2} & \mathbf{J} \boldsymbol{\Delta} \hat{\mathbf{p}}_{22}\left(k_{o}+\ell, k_{o}+\ell-1\right) \\
\mathbf{0}_{1 \times 2} & 0 & \mathbf{0}_{1 \times 2} & 1
\end{array}\right]}
\end{aligned}
$$

The measurement Jacobian now is given by (cf. (19)):

$$
\begin{aligned}
& \mathbf{H}_{k_{o}+\ell}= \\
& -\operatorname{Diag}\left(\left(\nabla \mathbf{h}_{k_{o}+\ell}^{(12)}\right) \mathbf{A}\left(\hat{\phi}_{1_{k_{o}+\ell \mid k_{o}+\ell-1}}\right),\left(\nabla \mathbf{h}_{k_{o}+\ell}^{(21)}\right) \mathbf{A}\left(\hat{\phi}_{2_{k_{o}+\ell \mid k_{o}+\ell-1}}\right)\right) \\
& \times\left[\begin{array}{cccc}
\mathbf{I}_{2} & \mathbf{J} \boldsymbol{\delta} \hat{\mathbf{p}}_{21}\left(k_{o}+\ell, k_{o}+\ell\right) & -\mathbf{I}_{2} & \mathbf{0}_{2 \times 1} \\
\mathbf{0}_{1 \times 2} & 1 & \mathbf{0}_{1 \times 2} & -1 \\
-\mathbf{I}_{2} & \mathbf{0}_{2 \times 1} & \mathbf{I}_{2} & \mathbf{J} \boldsymbol{\delta} \hat{\mathbf{p}}_{12}\left(k_{o}+\ell, k_{o}+\ell\right) \\
\mathbf{0}_{1 \times 2} & -1 & \mathbf{0}_{1 \times 2} & 1
\end{array}\right]
\end{aligned}
$$

Multiplication of (29) and (28) yields:

$$
\begin{aligned}
& \mathbf{H}_{k_{o}+\ell} \boldsymbol{\Phi}_{k_{o}+\ell-1} \cdots \boldsymbol{\Phi}_{k_{o}}= \\
& -\operatorname{Diag}\left(\left(\nabla \mathbf{h}_{k_{o}+\ell}^{(12)}\right) \mathbf{A}\left(\hat{\phi}_{1_{k_{o}}+\ell \mid k_{o}+\ell-1}\right),\right. \\
& {\left[\begin{array}{cccc}
\mathbf{I}_{2} & \mathbf{J} \boldsymbol{\Delta} \hat{\mathbf{p}}_{21}\left(k_{o}+\ell, k_{o}+\ell-1\right) & -\mathbf{I}_{2} & -\mathbf{J} \boldsymbol{\Delta} \hat{\mathbf{p}}_{22}\left(k_{o}+\ell, k_{o}+\ell-1\right) \\
\mathbf{0}_{1 \times 2} & 1 & \mathbf{0}_{1 \times 2} & \left.\mathbf{A}\left(\hat{\phi}_{k_{o}+\ell \mid k_{o}+\ell-1}\right)\right) \times \\
-\mathbf{I}_{2} & -\mathbf{J} \boldsymbol{\Delta} \hat{\mathbf{p}}_{11}\left(k_{o}+\ell, k_{o}+\ell-1\right) & \mathbf{I}_{2} & \mathbf{J} \boldsymbol{\Delta} \hat{\mathbf{p}}_{12}\left(k_{o}+\ell, k_{o}+\ell-1\right) \\
\mathbf{0}_{1 \times 2} & -1 & \mathbf{0}_{1 \times 2} & 1
\end{array}\right]}
\end{aligned}
$$

Thus, the observability matrix $\mathbf{M}$ (cf. (14)) can be written as:

$$
\begin{aligned}
& \mathbf{M}= \\
& \underbrace{-\operatorname{Diag}\left(\left(\nabla \mathbf{h}_{k_{o}}^{(12)}\right) \mathbf{A}\left(\hat{\phi}_{1_{k_{o} \mid k_{o}-1}}\right), \cdots,\left(\nabla \mathbf{h}_{k_{o}+m}^{(21)}\right) \mathbf{A}\left(\hat{\phi}_{2_{k_{o}+m \mid k_{o}+m-1}}\right)\right)}_{\mathbf{D}} \times \\
& {\left[\begin{array}{rrrr}
\mathbf{I}_{2} & \mathbf{J} \boldsymbol{\delta} \hat{\mathbf{p}}_{21}\left(k_{o}, k_{o}\right) & -\mathbf{I}_{2} & \mathbf{0}_{2 \times 1} \\
\mathbf{0}_{1 \times 2} & 1 & \mathbf{0}_{1 \times 2} & -1 \\
-\mathbf{I}_{2} & \mathbf{0}_{2 \times 1} & \mathbf{I}_{2} & \mathbf{J} \boldsymbol{\delta} \hat{\mathbf{p}}_{12}\left(k_{o}, k_{o}\right) \\
\mathbf{0}_{1 \times 2} & -1 & \mathbf{0}_{1 \times 2} & 1
\end{array}\right]} \\
& \underbrace{\mathbf{U}}_{\left.\begin{array}{rrrr}
\mathbf{I}_{2} & \mathbf{J} \boldsymbol{\Delta} \hat{\mathbf{p}}_{21}\left(k_{o}+1, k_{o}\right) & -\mathbf{I}_{2} & -\mathbf{J} \boldsymbol{\Delta} \hat{\mathbf{p}}_{22}\left(k_{o}+1, k_{o}\right) \\
\mathbf{0}_{1 \times 2} & 1 & \mathbf{0}_{1 \times 2} & -1 \\
-\mathbf{I}_{2} & -\mathbf{J} \boldsymbol{\Delta} \hat{\mathbf{p}}_{11}\left(k_{o}+1, k_{o}\right) & \mathbf{I}_{2} & \mathbf{J} \boldsymbol{\Delta} \hat{\mathbf{p}}_{12}\left(k_{o}+1, k_{o}\right) \\
\mathbf{0}_{1 \times 2} & -1 & \mathbf{0}_{1 \times 2} & 1 \\
\vdots & \vdots & \vdots & \vdots \\
\mathbf{I}_{2} & \mathbf{J} \boldsymbol{\Delta} \hat{\mathbf{p}}_{21}\left(k_{o}+m, k_{o}+m-1\right) & -\mathbf{I}_{2} & -\mathbf{J} \boldsymbol{\Delta} \hat{\mathbf{p}}_{22}\left(k_{o}+m, k_{o}+m-1\right) \\
\mathbf{0}_{1 \times 2} & 1 & \mathbf{0}_{1 \times 2} & \\
-\mathbf{I}_{2} & -\mathbf{J} \boldsymbol{\Delta} \hat{\mathbf{p}}_{11}\left(k_{o}+m, k_{o}+m-1\right) & \mathbf{I}_{2} & \mathbf{J} \boldsymbol{\Delta} \hat{\mathbf{p}}_{12}\left(k_{o}+m, k_{o}+m-1\right) \\
\mathbf{0}_{1 \times 2} & -1 & \mathbf{0}_{1 \times 2} & -1
\end{array}\right]}
\end{aligned}
$$

By proceeding similarly to the proof of Lemma 4.1, it can be verified (cf. [20]) that in general $\operatorname{rank}(\mathbf{M})=\operatorname{rank}(\mathbf{U})=4$. We thus see that the linearized error-state system model of the standard EKF-CL has different observability properties from that of the ideal EKF-CL. In particular, by processing the measurements collected in the time interval $\left[k_{o}, k_{o}+m\right]$, the EKF acquires information along the 4 directions of the state space corresponding to the observable subspace of the linearized system. However, the measurements actually provide information in only 3 directions of the state space (i.e., the robot-to-robot relative pose). This shows that the filter gains "spurious information" along unobservable directions of the underlying nonlinear CL system, which leads to inconsistency.

To probe further, we note that a basis of the right nullspace of $\mathbf{M}$ is given by (cf. (23)):

$$
\mathcal{N}(\mathbf{M})=\operatorname{span}\left[\begin{array}{ll}
\mathbf{n}_{1} & \mathbf{n}_{2}
\end{array}\right]
$$

Recall that these two vectors correspond to shifting the $x-$ $y$ plane, while the direction corresponding to the rotation is "missing" from the unobservable subspace of the EKF system model (cf. (23)). Therefore, we see that the filter will gain "nonexistent" information about the robots' global orientation. This will lead to an unjustified reduction in the orientation uncertainty, which will, in turn, further reduce the uncertainty in all state variables. We point out that the root cause of the problem is that the linearization points used for computing the Jacobians in the standard EKF-CL are the latest state estimates (i.e., the linearization point corresponding to the same state variable changes after each propagation). This increases the dimension of the observable subspace, and thus fundamentally alters the properties of the estimation process.

\section{Observability Constrained (OC)-EKF CL}

In the preceding section, it was shown that when the statepropagation Jacobians are evaluated using the latest state estimates, the EKF linearized error-state system model has an observable subspace of dimension higher than that of the actual CL system. This will always lead to unjustified reduction of the covariance estimates, and thus inconsistency. This issue is related to the problem of inconsistency of EKFSLAM, which was identified in [15]. In that work, to address the problem, we proposed an observability-based methodology for designing consistent estimators for EKF-SLAM. The key idea of this approach is to select linearization points to ensure that the EKF linearized error-state system model has appropriate observability properties, even at the cost of sacrificing linearization accuracy if necessary (i.e., choosing a linearization point which may increase the linearization error). By doing so, the influx of spurious information along the erroneously observable direction of the state space is avoided, thus improving the consistency of the estimates. It is important to note that this solution can be used for designing consistent estimators for any nonlinear system. Therefore, in this work, we adopt this observability-based methodology to design a consistent EKF estimator, termed Observability-Constrained $(O C)-E K F$ for CL. In particular, to guarantee the appropriate observability properties of the EKF linearized system model, we propose selecting the linearization points based on the following lemma:

Lemma 5.1: If the linearization points, $\mathbf{x}_{i_{k+1}}^{\star}, \mathbf{x}_{i_{k}}^{\star}$ and $\mathbf{x}_{j_{k}}^{\star}$, at which the filter Jacobian matrices $\boldsymbol{\Phi}_{i_{k}}=\boldsymbol{\Phi}_{i_{k}}\left(\mathbf{x}_{i_{k+1}}^{\star}, \mathbf{x}_{i_{k}}^{\star}\right)$ and $\mathbf{H}_{k}^{(i j)}=\mathbf{H}_{k}\left(\mathbf{x}_{i_{k}}^{\star}, \mathbf{x}_{j_{k}}^{\star}\right)$, are evaluated, are selected as

$$
\mathbf{x}_{i_{k+1}}^{\star}=\hat{\mathbf{x}}_{i_{k+1 \mid k}} \quad \mathbf{x}_{i_{k}}^{\star}=\hat{\mathbf{x}}_{i_{k \mid k-1}} \quad \mathbf{x}_{j_{k}}^{\star}=\hat{\mathbf{x}}_{j_{k \mid k-1}}
$$

then it is guaranteed that the EKF linearized error-state model has unobservable subspace of dimension 3. 
Proof: Using the linearization points (33), the statepropagation Jacobian $\boldsymbol{\Phi}_{i_{k}}$ (cf. (6)) is now computed as:

$$
\boldsymbol{\Phi}_{i_{k}}=\left[\begin{array}{cc}
\mathbf{I}_{2} & \mathbf{J}\left(\hat{\mathbf{p}}_{i_{k+1 \mid k}}-\hat{\mathbf{p}}_{i_{k \mid k-1}}\right) \\
\mathbf{0}_{1 \times 2} & 1
\end{array}\right]
$$

The difference compared to (6) in the standard EKF is that the robot position estimate prior to updating, $\hat{\mathbf{p}}_{i_{k \mid k-1}}$, is used in place of the updated estimate, $\hat{\mathbf{p}}_{i_{k \mid k}}$. In contrast, the measurement Jacobian, $\mathbf{H}_{k}^{(i j)}$, is computed in the same way as for the standard EKF (cf. (10)). As a result, using the definition of $\delta \hat{\mathbf{p}}_{i j}$ (26), the observability matrix of the OC-EKF becomes:

$$
\begin{aligned}
& \mathbf{M}^{\prime}= \\
& \underbrace{-\operatorname{Diag}\left(\left(\nabla \mathbf{h}_{k_{o}}^{(12)}\right) \mathbf{A}\left(\hat{\phi}_{1_{k_{o} \mid k_{o}-1}}\right), \cdots,\left(\nabla \mathbf{h}_{k_{o}+m}^{(21)}\right) \mathbf{A}\left(\hat{\phi}_{2_{k_{o}}+m \mid k_{o}+m-1}\right)\right)}_{\mathbf{D}}
\end{aligned}
$$

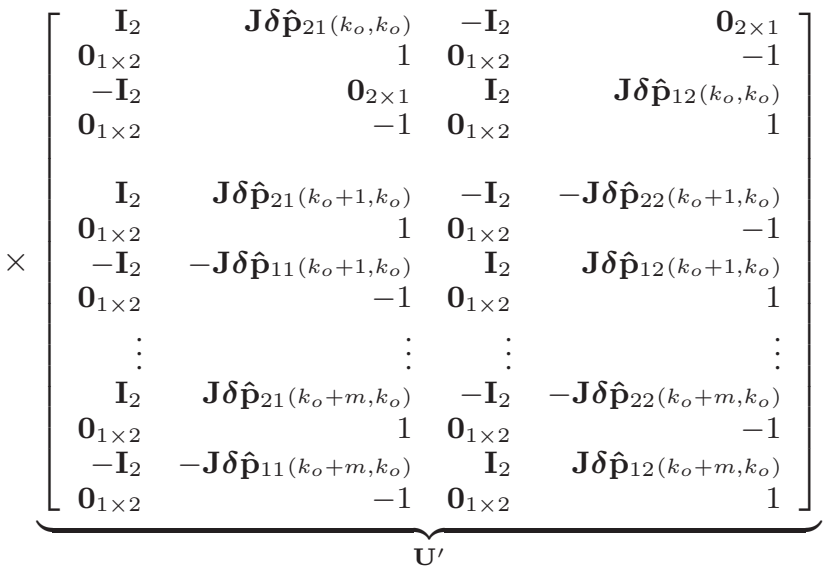

It becomes evident that, compared to the observability matrix of the ideal EKF-CL (cf. (22)), the only difference arising in $\mathbf{U}^{\prime}$ is that $\delta \mathbf{p}_{i j}$ is replaced by its estimate $\delta \hat{\mathbf{p}}_{i j}$, for $i, j=1,2$. Thus, by a proof analogous to that of Lemma 4.1, we can show that $\operatorname{rank}\left(\mathbf{M}^{\prime}\right)=3$. Moreover, the nullspace of $\mathbf{M}^{\prime}$ becomes:

$$
\mathcal{N}\left(\mathbf{M}^{\prime}\right)=\operatorname{span}\left[\begin{array}{cc}
\mathbf{I}_{2} & \mathbf{J} \hat{\mathbf{p}}_{1_{k_{o} \mid k_{o}-1}} \\
\mathbf{0}_{1 \times 2} & 1 \\
\mathbf{I}_{2} & \mathbf{J} \hat{\mathbf{p}}_{2_{k_{o} \mid k_{o}-1}} \\
\mathbf{0}_{1 \times 2} & 1
\end{array}\right]
$$

This implies that the error-state system model used by the OCEKF has unobservable subspace of dimension 3. The above proof is for the case of a two-robot team. In the more general case of an $N$-robot team ( $N \geq 2)$, it can be shown [20] that the corresponding observability matrix is of rank $3 N-3$, and thus its nullspace is also of dimension 3.

It is important to point out that, compared to the standard EKF, the only change in the OC-EKF is the way in which the state-propagation Jacobians are computed (cf. (34) vs. (6)), while the state estimate and covariance in the OC-EKF are propagated and updated in the same way as in the standard EKF. We also stress that the OC-EKF estimator is realizable and causal, as it does not require knowledge of the true state.

\section{Simulation Results}

A series of Monte-Carlo comparison studies were conducted under various conditions to validate the preceding theoretical analysis and the proposed OC-EKF. The metrics used to evaluate filter performance are: (i) the average root mean square (RMS) error, and (ii) the average normalized (state) estimation error squared (NEES) [10]. We compute these error metrics by averaging over all Monte Carlo runs for each time step. It is known that the NEES of an $M$-dimensional Gaussian random variable follows a $\chi^{2}$ distribution with $M$ degrees of freedom. Therefore, if a filter is consistent, we expect that the average NEES for each robot pose will be close to 3 for all $k$. The larger the deviation of the NEES from this value, the larger the inconsistency of the filter.

In the simulation tests, we consider the CL scenario in which four robots randomly move in an area of size $20 \mathrm{~m} \times 20 \mathrm{~m}$. 100 Monte Carlo simulations were performed, and during each run, all filters process the same data, to ensure a fair comparison. The three estimators compared are: (i) the ideal EKF, (ii) the standard EKF, and (iii) the OC-EKF.

For the results presented in this section, four identical robots with a simple differential drive model move on a planar surface, at a constant linear velocity of $v=0.25 \mathrm{~m} / \mathrm{sec}$, while the rotational velocity is drawn from the uniform distribution over $[-0.5,0.5] \mathrm{rad} / \mathrm{sec}$. The two drive wheels are equipped with encoders, which measure their revolutions and provide measurements of velocity (i.e., right and left wheel velocities, $v_{r}$ and $v_{l}$, respectively), with standard deviation equal to $\sigma=5 \% v$ for each wheel. These measurements are used to obtain linear and rotational velocity measurements for the robot, which are given by $v=\frac{v_{r}+v_{l}}{2}$ and $\omega=\frac{v_{r}-v_{l}}{a}$, where $a=0.5 \mathrm{~m}$ is the distance between the drive wheels. Thus, the standard deviations of the linear and rotational velocity measurements are $\sigma_{v}=\frac{\sqrt{2}}{2} \sigma$ and $\sigma_{\omega}=\frac{\sqrt{2}}{a} \sigma$, respectively.

Each robot records distance and bearing measurements to all other robots. Note that for simplicity we assume that each robot can observe all others at every time step. However, this is not a necessary assumption, as the analysis can easily be extended to the case where multiple propagation steps occur between updates (e.g., limited sensing range, or different sampling frequencies between proprioceptive and exteroceptive sensors). The standard deviation of the distance measurement noise is equal to $\sigma_{d}=0.1 \mathrm{~m}$, while the standard deviation of the bearing measurement noise is set to $\sigma_{\theta}=5 \mathrm{deg}$. It should be pointed out that the sensor-noise levels selected for the simulations are larger than what is typically encountered in practice. This was done purposefully, since larger noise levels lead to higher estimation errors, which make the effects of inconsistency more apparent.

The results of all filters for one of the robots are presented in Fig. 1 (the results for the other three robots are very similar and are omitted due to space limitations). Specifically, Fig. 1(a) shows the orientation estimation errors, obtained from a typical simulation. Clearly, the standard-EKF errors grow significantly faster than those of the ideal and the OC-EKF, which indicates that the standard EKF tends to diverge. Note also that although the orientation errors of the ideal and the OC-EKF remain well within their corresponding $3 \sigma$ bounds, those of the standard EKF exceed them. Most importantly, in contrast to those of the 


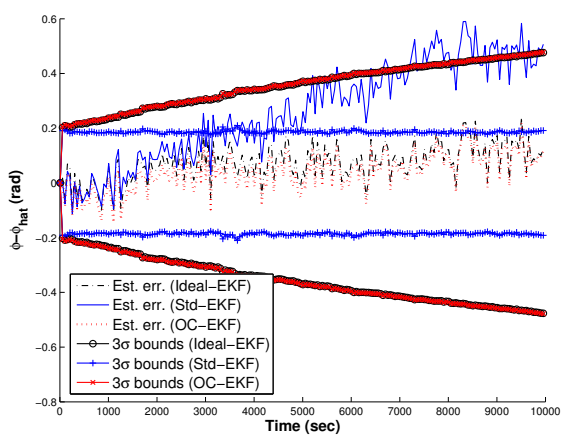

(a)

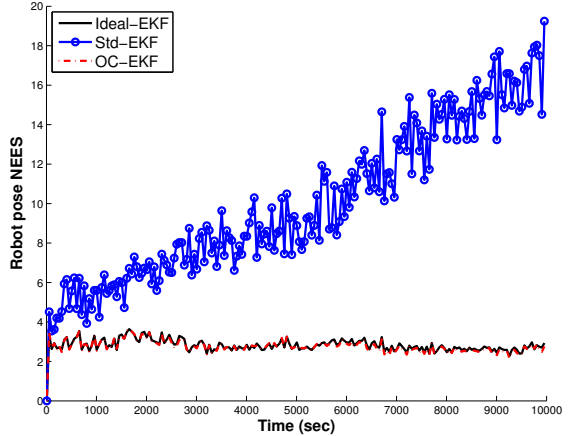

(b)
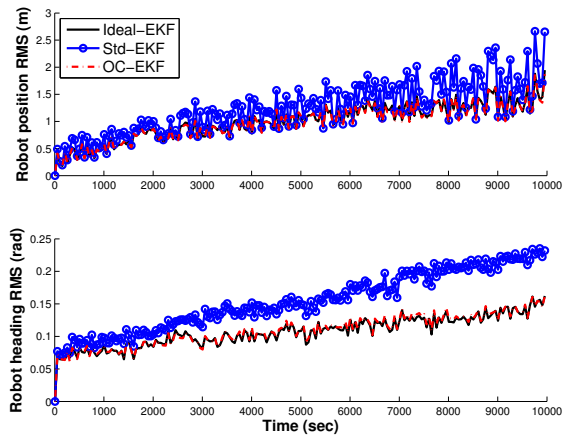

(c)

Fig. 1. Simulation results for one robot of a 4-robot team. The results for the other robots are similar to the ones presented here. (a) Orientation estimation errors vs. $3 \sigma$ bounds obtained from one typical realization of the Monte Carlo simulations. The $\sigma$ values are computed as the square-root of the corresponding element of the estimated covariance matrix. (b)-(c) Monte Carlo results of average NEES and RMS errors, respectively. In these two plots, the solid lines correspond to the ideal EKF, the solid lines with circles to the standard EKF, and the dash-dotted lines to the OC-EKF. Note that the NEES and RMS errors as well as the estimation errors of the ideal and the OC-EKF are almost identical, which makes the corresponding lines difficult to distinguish.

OC-EKF, the $3 \sigma$ bounds of the standard EKF remain almost constant as if the orientation of the robot was observable. However, as discussed in Section IV, the robots have no access to absolute orientation information and thus the orientation covariance should continuously grow (as is the case for the ideal and the OC-EKF). The results of Fig. 1(a) clearly demonstrate that the incorrect observability properties of the standard EKF cause an unjustified reduction of the orientation uncertainty.

Figs. 1(b)-1(c) show the average NEES and RMS errors. As evident, the performance of the OC-EKF is almost identical to that of the ideal EKF, and substantially better than the standard EKF, both in terms of RMS errors and NEES. This occurs even though the OC-EKF Jacobians are less accurate than those of the standard EKF, as explained in the preceding section. This fact indicates that the errors introduced by the use of inaccurate linearization points (for computing the Jacobians) have a less detrimental effect on consistency than the use of an error-state system model with observable subspace of dimension larger than that of the actual CL system.

\section{EXPERIMENTAL RESULTS}

A real-world experiment was performed to further validate the presented analysis and the OC-EKF algorithm. During the test, a team of four Pioneer I robots moves in a rectangular area of $2.5 \mathrm{~m} \times 4.5 \mathrm{~m}$, within which the positions of the robots are tracked by an overhead camera. The vision system provides measurements of the robot poses in a global coordinate frame, which serve as the ground truth for evaluating the estimators' performance in terms of NEES and RMS errors. The standard deviation of the noise in these measurements is approximately $0.5 \mathrm{deg}$ for orientation and $0.01 \mathrm{~m}$, along each axis, for position. The robots were commanded to move at a constant velocity of $v=0.1 \mathrm{~m} / \mathrm{sec}$ while avoiding collision with the boundaries of the arena as well as with their teammates. In order to preserve legibility, only the first $200 \mathrm{sec}$ of the four robot trajectories are shown in Fig. 2(a).
Although four robots of the same model were used, their wheel-encoders are not equally accurate. Specifically, during calibration, the velocity measurement errors, modeled as zero-mean white Gaussian noise processes, exhibited standard deviations ranging from $\sigma_{v_{\min }}=3.8 \% v$ for the most accurate odometer to $\sigma_{v_{\max }}=6.9 \% \mathrm{v}$ for the robot with the highest noise levels. Similarly, the standard deviations of the rotational velocity measurements assumed values between $\sigma_{\omega_{\min }}=0.0078 \mathrm{rad} / \mathrm{sec}$ and $\sigma_{\omega_{\max }}=0.02 \mathrm{rad} / \mathrm{sec}$ for the four robots. We observe that as a result of this variability of sensor characteristics, the experiment can be considered as involving a heterogeneous robot team.

Distance-bearing measurements are produced synthetically using the differences in the positions of the robots, as these are recorded by the overhead camera, expressed in the measuring robot's coordinate frame, with the addition of noise. For the experimental results shown in this section, the distance and bearing measurements are corrupted by zero-mean white Gaussian noise, with standard deviation $\sigma_{d}=0.05 \mathrm{~m}$ for distance and $\sigma_{\theta}=2 \mathrm{deg}$ for bearing measurements, respectively. Pose estimation was run offline, and two filters were compared: (i) the standard EKF, and (ii) the proposed OC-EKF. Comparative results of the standard EKF and the proposed OC-EKF for one of the robots are presented in Figs. 2(b) and 2(c). The results for the other robots are similar to the ones presented here. From the experimental results it becomes clear that, just as in simulation, also in the real-world experiment the OC-EKF outperforms the standard EKF, in terms of both accuracy and consistency. Moreover, these results further support our conjecture that the mismatch in the dimension of the unobservable subspace between the linearized CL system and the underlying nonlinear system is a fundamental cause of filter inconsistency.

\section{CONCLUSIONS}

In this paper, we have studied in depth the issue of consistency in EKF-based CL, from an observability perspective. By 


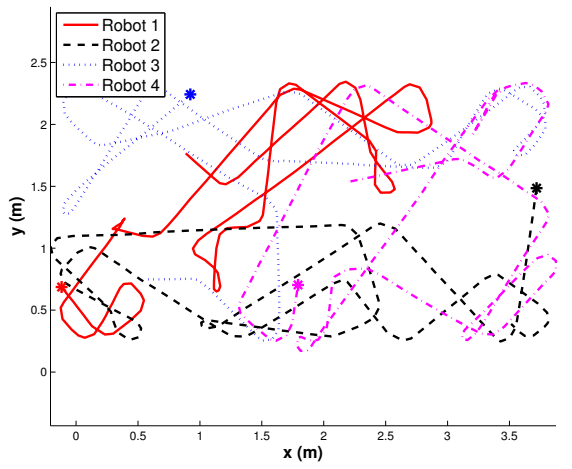

(a)

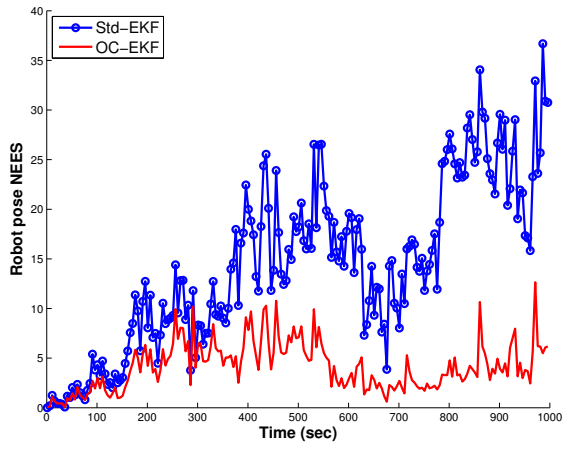

(b)
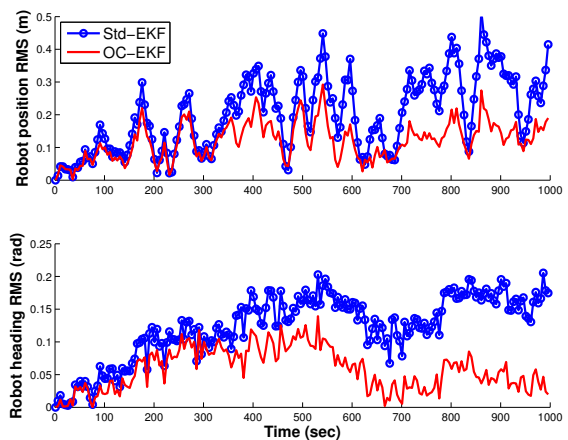

(c)

Fig. 2. Experimental results: (a) Four Pioneer I robots move inside a $2.5 \mathrm{~m} \times 4.5 \mathrm{~m}$ arena, with starting positions marked by $*$. To maintain clarity, only the first $200 \mathrm{sec}$ of the trajectories are plotted. (b)-(c) NEES and RMS errors respectively for one robot. The results for the other robots are similar to the ones presented here. As in the simulations, the OC-EKF outperforms the standard EKF in terms of both accuracy (RMS errors) and consistency (NEES).

comparing the observability properties of the linearized errorstate model employed in the EKF with those of the underlying nonlinear CL system, we proved that the observable subspace of the standard EKF-CL is always of higher dimension than that of the actual CL system. As a result, the covariance estimates of the EKF undergo reduction in directions of the state space where no information is available, and thus the standard EKF-CL is always inconsistent. Moreover, we proposed a new OC-EKF, which improves the consistency of EKF-based CL. The design methodology followed for deriving the OC-EKF requires appropriate selection of the linearization points at which the Jacobians are evaluated, which ensures that the observable subspace of the linearized error-state system model is of the same dimension as that of the underlying actual system. Simulations and experiments have verified that the proposed OC-EKF performs better, in terms of both accuracy and consistency, than the standard EKF.

\section{ACKNOWLEDGEMENTS}

This work was supported by the University of Minnesota (DTC), the National Science Foundation (IIS-0643680, IIS0811946, IIS-0835637), and the University of California, Riverside (BCOE).

\section{REFERENCES}

[1] R. Kurazume and S. Hirose, "An experimental study of a cooperative positioning system,” Auto. Rob., vol. 8, no. 1, pp. 43-52, Jan. 2000.

[2] I. M. Rekleitis, G. Dudek, and E. E. Milios, "Multi-robot cooperative localization: a study of trade-offs between efficiency and accuracy," in Proc. of the IEEE/RSJ Int. Conf. on Intel. Rob. and Sys., Lausanne, Switzerland, Sept. 30-Oct. 4, 2002, pp. 2690-2695.

[3] S. I. Roumeliotis and G. A. Bekey, "Distributed multirobot localization," IEEE Trans. Rob. Autom., vol. 18, no. 5, pp. 781-795, Oct. 2002.

[4] A. Howard, M. J. Mataric, and G. S. Sukhatme, "Localization for mobile robot teams using maximum likelihood estimation," in Proc. of the IEEE/RSJ Int. Conf. on Intel. Rob. and Sys., Lausanne, Switzerland, Sept. 30-Oct. 4, 2002, pp. 434-439.

[5] E. D. Nerurkar, S. I. Roumeliotis, and A. Martinelli, "Distributed maximum a posteriori estimation for multi-robot cooperative localization," in Proc. of the IEEE Int. Conf. on Rob. and Autom., Kobe, Japan, May 12-17, 2009, pp. 1402-1409.

[6] D. Fox, W. Burgard, H. Kruppa, and S. Thrun, "A probabilistic approach to collaborative multi-robot localization," Auto. Rob., vol. 8, no. 3, pp. 325-344, June 2000.
[7] S. Panzieri, F. Pascucci, and R. Setola, "Multirobot localization using interlaced extended Kalman filter," in Proc. of the IEEE/RSJ Int. Conf. on Intel. Rob. and Sys., Beijing, China, Oct. 9-15, 2006, pp. 2816-2821.

[8] N. Karam, F. Chausse, R. Aufrere, and R. Chapuis, "Localization of a group of communicating vehicles by state exchange," in Proc. of the IEEE/RSJ Int. Conf. on Intel. Rob. and Sys., Beijing, China, Oct. 9-15, 2006, pp. 519-524.

[9] A. Martinelli, "Improving the precision on multi robot localization by using a series of filters hierarchically distributed," in Proc. of the IEEE/RSJ Int. Conf. on Intel. Rob. and Sys., San Diego, CA, Oct. 29Nov. 2, 2007, pp. 1053-1058.

[10] Y. Bar-Shalom, X. Li, and T. Kirubarajan, Estimation with applications to tracking and navigation. New York: Wiley, 2001.

[11] S. Julier and J. Uhlmann, "A counter example to the theory of simultaneous localization and map building," in Proc. of the IEEE Int. Conf. on Rob. and Autom., Seoul, Korea, May 21-26, 2001, pp. 4238-4243.

[12] J. Castellanos, J. Neira, and J. Tardos, "Limits to the consistency of EKF-based SLAM," in Proc. of the 5th IFAC Symp. on Intel. Auto. Veh., Lisbon, Portugal, July 5-7, 2004, pp. 1244-1249.

[13] T. Bailey, J. Nieto, J. Guivant, M. Stevens, and E. Nebot, "Consistency of the EKF-SLAM algorithm," in Proc. of the IEEE/RSJ Int. Conf. on Intel. Rob. and Sys., Beijing, China, Oct. 9-15, 2006, pp. 3562-3568.

[14] S. Huang and G. Dissanayake, "Convergence and consistency analysis for extended Kalman filter based SLAM," IEEE Trans. Rob., vol. 23, no. 5, pp. 1036-1049, Oct. 2007.

[15] G. P. Huang, A. I. Mourikis, and S. I. Roumeliotis, "Analysis and improvement of the consistency of extended Kalman filter-based SLAM," in Proc. of the IEEE Int. Conf. on Rob. and Autom., Pasadena, CA, May 19-23, 2008, pp. 473-479.

[16] A. Bahr, M. R. Walter, and J. J. Leonard, "Consistent cooperative localization," in Proc. of the IEEE Int. Conf. on Rob. and Autom., Kobe, Japan, May 12-17, 2009, pp. 3415-3422.

[17] A. Howard, M. J. Mataric, and G. S. Sukhatme, "Putting the 'i' in 'team': an ego-centric approach to cooperative localization," in Proc. of the IEEE Int. Conf. on Rob. and Autom., Taipei, Taiwan, Sept. 14-19, 2003, pp. 868-874.

[18] A. Martinelli and R. Siegwart, "Observability analysis for mobile robot localization," in Proc. of the IEEE/RSJ Int. Conf. on Intel. Rob. and Sys., Alberta, Canada, Aug. 2-6, 2005, pp. 1471-1476.

[19] P. Maybeck, Stochastic Models, Estimation and Control. Academic Press, 1979, vol. 1.

[20] G. P. Huang and S. I. Roumeliotis, "On the consistency of multirobot cooperative localization," MARS Lab, University of Minnesota, Minneapolis, MN, Tech. Rep., Jan. 2009. [Online]. Available: www.cs.umn.edu/ ghuang/paper/TR_CL_Consistency.pdf

[21] C. Meyer, Matrix Analysis and Applied Linear Algebra. SIAM, 2001. 